\title{
Simulação de alta fidelidade realística para o ensino de emergências médicas na prática odontológica: relato de experiência
}

\author{
Rogério Heládio Lopes Motta*; Juliana Cama Ramacciato*; Luciana Butini Oliveira**; Marcelo \\ Amade Camargo***; Renato Nardi Pedro***; Roberto Salvador Martins***; Rogério Fortunato de \\ Barros***
}

* Docente, Área de Farmacologia, Anestesiologia e Terapêutica, Faculdade São Leopoldo Mandic

** Docente, Área de Odontopediatria, Faculdade São Leopoldo Mandic

*** Docente, Curso de Medicina, Faculdade São Leopoldo Mandic

Recebido em 31/08/2017. Aprovado em 09/01/2018.

\begin{abstract}
RESUMO
Os cirurgiões-dentistas devem estar preparados para eventuais situações de emergências médicas que podem ocorrer durante o atendimento odontológico. Entretanto, muitos profissionais não se sentem preparados para executar estes procedimentos e têm pouca experiência em treinamentos práticos. Desta forma, o objetivo deste artigo é relatar a experiência de uma atividade de simulação em diferentes cenários de emergências médicas na prática odontológica. Esta metodologia envolve o uso de um simulador de alta fidelidade e permite a discussão na sala de debriefing sobre os principais procedimentos e treinamento prático para o manejo de emergências médicas. A simulação de alta fidelidade realística para o ensino de emergências médicas na prática odontológica representa um cenário inovador e é eficaz para o processo de ensino e aprendizagem.

Descritores: Educação em Odontologia. Assistência Odontológica. Emergências. Simulação por Computador.
\end{abstract}

\section{INTRODUÇÃO}

Com o avanço da Medicina e consequente aumento da expectativa de vida da população, maior é a diversidade de pacientes que procuram serviços odontológicos ${ }^{1}$. Desta forma, o cirurgião-dentista deve estar apto a manejar 
intercorrências que muitas vezes não estão vinculadas apenas aos procedimentos odontológicos, como também as alterações sistêmicas pré-existentes de seus pacientes ${ }^{2}$.

Adicionalmente, a lei brasileira 5081/66 3 que regula o exercício da Odontologia, apresenta em seu artigo $6^{\circ}$ inciso VIII que compete ao cirurgião-dentista "prescrever e aplicar medicação de urgência no caso de acidentes graves que comprometam a vida e a saúde do paciente".

Embora o assunto seja de extrema importância, estudos têm demonstrado que o cirurgião-dentista nem sempre se sente preparado e seguro para lidar com uma emergência médica. Um recente estudo realizado por Alkandari et al. ${ }^{4}$ demonstrou que os cirurgiões-dentistas avaliados possuíam conhecimento inadequado em relação às manobras de ressuscitação cardiopulmonar (RCP), sendo que apenas $36 \%$ dos participantes da pesquisa mostraram conhecimento adequado sobre o assunto. Arsati et al. ${ }^{5}$ entrevistaram 498 dentistas brasileiros em relação a seu preparo para lidar com emergências médicas em ambiente odontológico. Os autores constataram que $79,7 \%, 72,9 \%$ e $85,7 \%$ dos profissionais não se sentiam capazes de lidar com situações graves como infarto do miocárdio, anafilaxia e acidentes vasculares encefálicos (AVE), respectivamente. Além disso, quase $60 \%$ da amostra afirmou que não sabia realizar as manobras de RCP, seja pela falta de aprendizado da técnica durante o curso de graduação ou de informações atualizadas sobre o tema.

Este tema também tem sido estudado com graduandos de Odontologia para avaliar o conhecimento e habilidades em relação ao suporte básico de vida e RCP. Um estudo realizado por Le et al. ${ }^{6}$ enfatizou a necessidade de incorporar exercícios periódicos de simulação com o intuito de aumentar a habilidade do graduando para manejar situações de emergências médicas. Breuer et al. ${ }^{7}$ apontaram dificuldades do estudante de Odontologia sobre o tema e concluíram que o treinamento para suporte básico de vida em uma base regular é necessário e deve ser inserido de maneira padronizada no conteúdo programático de emergências médicas para estudantes de Odontologia.

As Diretrizes Curriculares Nacionais (DCN) para os Cursos de Odontologia estabelecem no Art. $4^{\circ}$ que "o trabalho dos profissionais de saúde deve ser fundamentado na capacidade de tomar decisões visando o uso apropriado, eficácia e custo-efetividade da força de trabalho, de medicamentos, de equipamentos, de procedimentos e de práticas" ${ }^{\text {. As DCN }}$ também preconizam para o processo de ensinoaprendizagem a organização de conteúdos e inserção de metodologias inovadoras.

Novas estratégias e recursos estão sendo apresentados e disponibilizados nas instituições de ensino com o intuito do aprimoramento do aprendizado do estudante em relação a emergências médicas em Odontologia. Dentre elas, destaca-se o uso de métodos de simulação de alta fidelidade realística. Um trabalho realizado por Newby et al. ${ }^{9}$ comprovou que o treinamento realístico no gerenciamento de emergências médicas para estudantes de Odontologia pode ser um método eficaz para o aprendizado. Adicionalmente, este modo de treinamento poderia beneficiar os alunos se incorporados em cursos de graduação de Odontologia.

Em relação à simulação de alta fidelidade realística são escassos os estudos que avaliaram esta metodologia e recurso para o ensino de emergências médicas na prática odontológica. Os resultados apontam que a simulação de alta fidelidade é eficaz no processo de ensinoaprendizagem e para o preparo de estudantes para o manejo de emergências médicas ${ }^{10-12}$. 
Embora o tema seja importante, o modelo de simulação realística de alta fidelidade como estratégia de ensino sobre o tema ainda é recente no Brasil, o que direcionou o planejamento e a realização desta atividade. Com base no exposto, o objetivo deste artigo é relatar a experiência de uma atividade de simulação com diferentes cenários de emergências médicas na prática odontológica.

\section{RELATO DE EXPERIÊNCIA}

Este é um relato de experiência de uma atividade didática desenvolvida por uma equipe multidisciplinar de professores (médicos, cirurgiões-dentistas e um enfermeiro) para o ensino e aprimoramento de competências sobre o manejo de situações de emergências médicas em ambiente odontológico. O planejamento da atividade teve início em outubro de 2016, com reuniões periódicas entre os profissionais visando o aprimoramento do enfoque teórico e prático das simulações de emergências médicas em ambiente odontológico considerando as recomendações legais vigentes. Em abril e maio de 2017 foram realizados testes para realizar os ajustes finais e as atividades foram realizadas em junho e agosto de 2017.

A proposta da simulação realística de alta fidelidade é possibilitar o treinamento em ambiente simulado com atividades guiadas que visam reproduzir aspectos e detalhes de situações reais de forma interativa, e a sua fidelidade depende do quanto este consegue se aproximar da realidade da prática clínica. Dentro deste contexto, para a realização das atividades foram elaborados casos clínicos hipotéticos, assim como a caracterização do ambiente com equipo, mocho e instrumentais clínicos para que o aluno pudesse se sentir em um ambiente odontológico (figura 1).

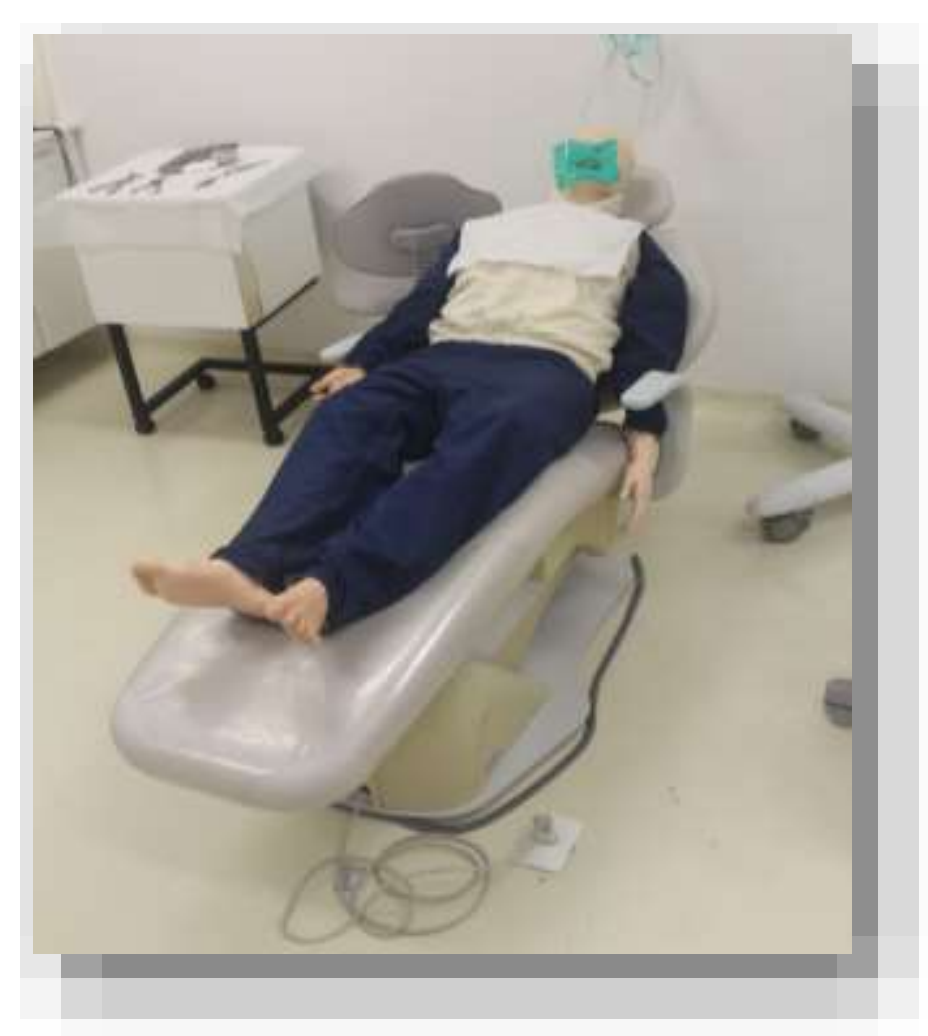

Figura 1. Robô de simulação posicionado em cadeira odontológica 


\section{Local da atividade}

A atividade foi desenvolvida e realizada no Laboratório de Simulação da Faculdade São Leopoldo Mandic. Esta unidade é equipada com robôs de simulação, manequins, sala de controle de recursos audiovisuais e duas salas de debriefing que possibilitam a discussão de cada cenário de simulação realizado com os alunos. Adicionalmente, estas salas possuem um sistema audiovisual integrado com as salas de simulação que permitem que os alunos que não estejam no cenário de simulação possam assistir por diferentes ângulos de filmagem todo a sequência de cada caso clínico simulado (figura 2).

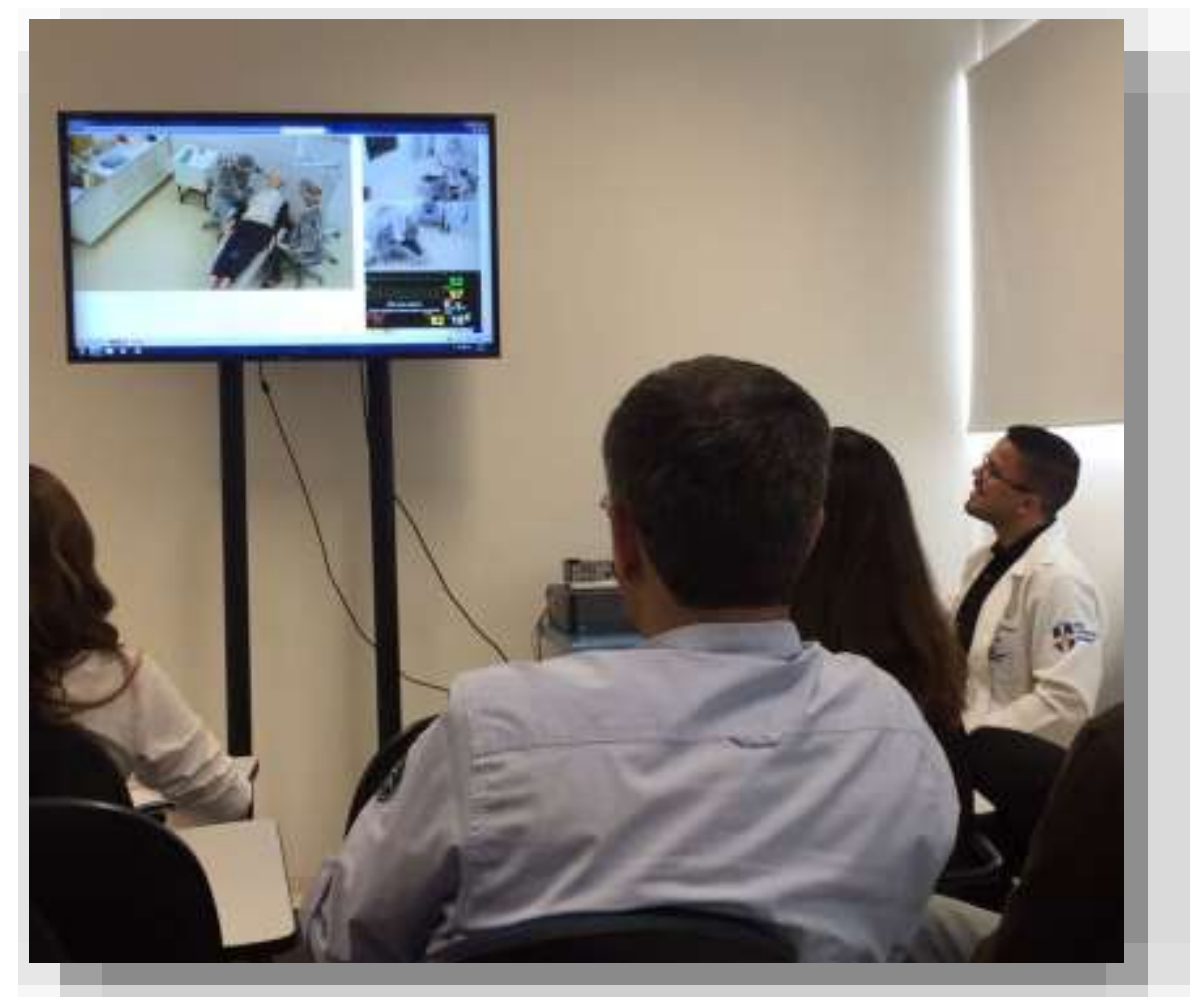

Figura 2. Visualização do cenário de simulação por meio de monitor da sala de debriefing para acompanhamento das simulações

\section{Participantes}

Em cada sessão da atividade participaram cirurgiões-dentistas de diferentes especialidades, totalizando 12 profissionais em cada sessão. Os profissionais foram divididos em duplas para a atividade da simulação, e no total foram realizados 6 cenários de simulação envolvendo possíveis intercorrências clínicas na cadeira odontológica.

\section{Instrutores}

Três médicos participaram da atividade como instrutores, um enfermeiro e dois cirurgiões-dentistas acompanharam os demais alunos na sala de debriefing. Um instrutor permaneceu no mesmo ambiente do cenário simulado em todos os casos propostos, enquanto outro instrutor permaneceu na sala de controle de recursos audiovisuais (figura 3 ). $\mathrm{O}$ terceiro 
instrutor acompanhou as duplas na apresentação de cada caso proposto.

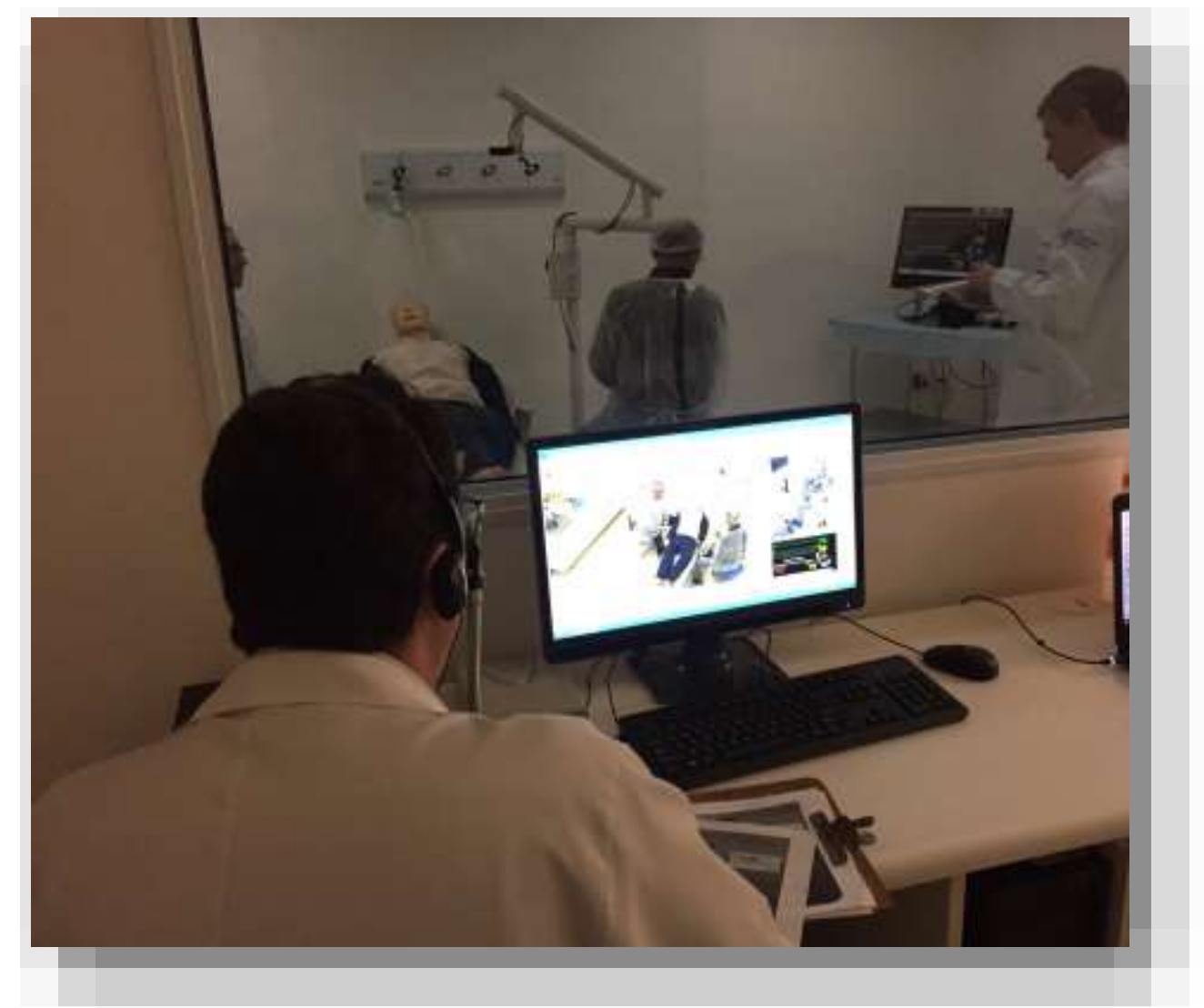

Figura 3. Sala de controle de recursos audiovisuais

\section{Simulações}

Cada sessão da atividade proposta foi realizada em um dia, com aulas teóricas no período da manhã e atividades de simulação no período da tarde. As aulas teóricas foram compostas por temas relacionados à avaliação pré-clínica do paciente odontológico, além da prevenção e manejo de possíveis situações de emergências médicas no atendimento odontológico.

Para as atividades de simulação foram utilizados diferentes cenários com um simulador de alta fidelidade (Resusci Anne Simulator, Laerdal ${ }^{\circledR}$ Gatesville, Texas, EUA). Cada cenário idealizado envolveu o atendimento odontológico de um paciente com diferentes características sistêmicas (diabético, hipertenso ou asmático, por exemplo), além de diferentes procedimentos clínicos (cirurgia de instalação de implantes, exodontias ou tratamento endodôntico, por exemplo). Embora estes cenários apresentassem diferentes situações hipotéticas em ambiente odontológico, (como por exemplo uma crise aguda de asma), todos eles tiveram as mesmas propostas básicas:

a. Identificar possíveis riscos do atendimento odontológico do paciente mediante a realização de uma avaliação pré-clínica rigorosa;

b. Analisar as competências individuais de 
cada participante mediante uma situação simulada de emergência médica em ambiente odontológico;

c. Acompanhar o desempenho de cada participante de forma individualizada para posterior discussão em ambiente de debriefing;

d. Discutir os procedimentos realizados pelos participantes e detectar as possíveis falhas e dificuldades dos mesmos no cenário simulado;

e. Ressaltar as condutas adequadas realizadas pelos participantes;

f. Sugerir possíveis melhorias para eventuais casos similares que possam ocorrer, relacionando-os com possíveis casos reais dos participantes.

$\mathrm{O}$ instrutor que permaneceu em cada cenário avaliou o procedimento realizado por meio de um checklist, e eventuais dúvidas e dificuldades apresentadas foram anotadas para serem discutidas na sala de debriefing. $\mathrm{O}$ instrutor que permaneceu na sala de controle de recursos audiovisuais acompanhou cada cenário de modo a reproduzir a voz do paciente e as possíveis manifestações sistêmicas de cada cenário proposto (dificuldade respiratória, por exemplo). O terceiro instrutor acompanhou cada dupla de forma efetiva, seja para esclarecer alguma dúvida do perfil clínico do paciente apresentado, bem como atuando como um membro fictício da família do paciente para responder possíveis dúvidas sobre sua história médica. Os instrutores não passaram informações técnicas em nenhum momento da simulação, com o intuito de não comprometer a performance de cada aluno.

Cada cenário foi programado para durar até 10 minutos, e após a realização de cada simulação foi realizada uma discussão de 20 minutos na sala de debriefing. A equipe elaborou diferentes checklists padronizados para facilitar a dinâmica de cada discussão, sendo que primeiramente cada dupla descrevia o cenário realizado e eventuais dificuldades apresentadas, para então os instrutores realizarem uma dinâmica com os demais alunos.

No final do dia foi realizada uma última discussão geral sobre a proposta apresentada, e foi requisitado para que cada participante pudesse relatar a sua percepção sobre a atividade. Com o objetivo de detalhar este relato de experiência, algumas opiniões de diferentes profissionais são apresentadas a seguir.

"Uma atividade que nos remete a realidade clínica de uma situação de emergência médica de uma maneira real. Mais simulações de outras situações clínicas poderiam ter sido realizadas. Eu super indico para outros profissionais."

"A atividade foi muito além das expectativas, pois o enfoque é voltado para prática clínica diária, com a experiência da área médica e da área odontológica. A simulação sem dúvida é um diferencial, pois a aplicação do que se aprendeu é direta, e nesse momento as dicas e informações fazem a diferença. Atividade de excelência que todo dentista deveria fazer frequentemente."

"A melhor atividade que já fiz sobre o tema, e a simulação de emergências proporcionou um grande aprendizado. Considerando o conteúdo programático, acredito que poderia ser acrescentado mais 8 horas."

"Tive a oportunidade de vivenciar tomadas de decisões consequentes de sinais $e$ sintomas avaliados a partir de uma simulação clínica de intercorrência. Após esta atividade pude averiguar a maior necessidade do cirurgião-dentista reciclar e adquirir conhecimentos necessários para o atendimento de situações de emergências 
médicas em ambiente odontológico."

\section{CONSIDERAÇÕES FINAIS}

As atividades de simulação podem ser consideradas metodologias ativas que reúnem bases teóricas e práticas. Além disso, representam um cenário inovador de ensino e aprendizagem com aspectos positivos para a formação e treinamento em Odontologia, contemplando princípios fundamentais das Diretrizes Curriculares do Curso de Odontologia.

De acordo com um dos princípios da clínica ampliada ${ }^{13,14}$, é necessário desenvolver no profissional da saúde um compromisso radical com o sujeito doente, visto de modo singular; assumir a responsabilidade sobre os usuários dos serviços de saúde; buscar ajuda multidisciplinar e em outros setores. Adicionalmente, os profissionais da área da saúde precisam reconhecer os limites dos conhecimentos e das tecnologias empregadas na prática clínica.

Por meio do presente relato é possível inferir que a simulação de alta fidelidade é uma estratégia eficaz para treinamento de indivíduos e equipes em capacidades técnicas (conhecimentos e habilidades), além de beneficiar a comunicação, atitudes e trabalho em equipe. Esta metodologia também é valorizada pelos alunos por aumentar a autoconfiança, percepção cognitiva e psicomotora para o manejo de emergências médicas no consultório odontológico que podem despertar situações de muita apreensão.

Há uma tendência crescente de centros e laboratórios de simulação no Brasil. Entretanto, como limitações, destacam-se os custos elevados para a criação da infraestrutura física $\mathrm{e}$ treinamentos dos profissionais para o emprego destes recursos tecnológicos.

Com base no exposto, pode-se concluir que métodos de simulação de alta fidelidade realística para o ensino de emergências médicas na prática odontológica representam um cenário inovador para o processo de ensino e aprendizagem, tendo alunos e professores como protagonistas.

\section{ABSTRACT \\ High-fidelity simulation as a teaching method in medical emergencies in dental practice: experience report}

Dentists should be prepared to manage eventual medical emergencies that occur in the dental practice. However, many dentists are not fully prepared to manage these situations and have insufficient experience training in medical emergencies. The aim of this paper is to report the experience of educational activities using a high-fidelity simulator in different scenarios in order to prepare dental professionals for medical emergencies in the dental clinic. This methodology allows discussion in the debriefing room regarding the main techniques and procedures to be performed in clinical practice in cases of emergencies and training of dentists to meet these situations. It can be concluded that high-fidelity simulation represents an innovative learning scenario and it is an effective educational method to prepare dental professionals for medical emergencies.

Descriptors: Dental Education. Dental Care. Emergencies. Computer Simulation.

\section{REFERÊNCIAS}

1. Caputo IG, Bazzo CJ, Silva RH, Júnior ED. Vidas em Risco: Emergências Médicas em consultório odontológico. Rev Cir Traumatol Buco-Maxilo-Fac. 2010;10 (3):51-8.

2. Malamed SF. Knowing your patients. J Am Dent Assoc. 2010;141(1):3-7.

3. Brasil. Lei n ${ }^{\circ}$ 5081, de 24 de agosto de 1966. Regulamenta o exercício da Odontologia no Brasil. Brasília, Diário Oficial da União, 1966.

4. Alkandari SA, Alyahya L, Abdulwahab M. 
Cardiopulmonary resuscitation knowledge and attitude among general dentists in Kuwait. World J Emerg Med. 2017;8(1):1924.

5. Arsati F, Montalli VA, Flório FM, Ramacciato JC, da Cunha FL, Cecanho R, et al. Brazilian dentists' attitudes about medical emergencies during dental treatment. J Dent Educ. 2010;74(6):661-6.

6. Le TT, Scheller EL, Pinsky HM, Stefanac SJ, Taichman RS. Ability of dental students to deliver oxygen in a medical emergency. $\mathrm{J}$ Dent Educ 2009;73(4):499-508.

7. Breuer G, Knipfer C, Huber T, Huettl S, Shams N, Knipfer K, et al. Competency in managing cardiac arrest: a scenario-based evaluation of dental students. Acta Odontol Scand. 2016;74(4):241-9.

8. Brasil. Conselho Nacional de Educação. Diretrizes Curriculares Nacionais do curso de graduação em Odontologia. Resolução CNE/CES 3/2002. Diário Oficial da União, Brasília, 4 de março de 2002. Seção 1, p. 10.

9. Newby JP, Keast J, Adam WR. Simulation of medical emergencies in dental practice: development and evaluation of an undergraduate training programme. Aust Dent J. 2010 Dec;55(4):399-404.

10. Tan GM. A medical crisis management simulation activity for pediatric dental residents and assistants. J Dent Educ. 2011;75:782-90.

11. Bilich LA, Jackson SC, Bray BS, Wilson MN. High-fidelity simulation: preparing dental hygiene students for managing medical emergencies. J Dent Educ. 2015;79:1074-81.
12. Roy E, VE Quinsat VE, Bazin O, Lesclous P, Lejus-Bourdeau C. High-fidelity simulation in training dental students for medical life-threatening emergency. Eur J Dent Educ. 2017; Aug 23. doi: 10.1111/eje. 12284.

13. Brasil. Ministério da Saúde. Secretaria de Atenção à Saúde. Núcleo técnico da Política Nacional de Humanização. Clínica Ampliada, Equipe de referência e Projeto Terapêutico Singular/Ministério da Saúde, Secretaria de Atenção à Saúde, Núcleo técnico da Política Nacional de Humanização. 2. ed. - Brasília: Editora do Ministério da Saúde, 2008.

14. Brasil. Ministério da Saúde. Secretaria de Atenção à Saúde. Política Nacional de Humanização da Atenção e Gestão do SUS. Clínica ampliada e Compartilhada / Ministério da Saúde, Secretaria de Atenção à Saúde, Política Nacional de Humanização da Atenção e Gestão do SUS. - Brasília: Ministério da Saúde, 2009.

\section{Correspondência para:}

Rogério Heládio Lopes Motta

e-mail: rogerio.motta@slmandic.edu.br

Rua José Rocha Junqueira, 13

13045-755 Campinas/ SP 